\title{
3D SOM Neighborhood Algorithm
}

\author{
Hongsong Li, Fulin Cheng, Yanhua Wang, Xinyu Ai \\ School of Information and Communication \\ Guilin University of Electronic Technology \\ Guilin, Guangxi, China \\ hongsongli@guet.edu.cn, guetcfl@163.com
}

\begin{abstract}
Neighborhood algorithm is an important part of 3D SOM algorithm. We proposed three kinds of neighborhood shape and two kinds of neighborhood decay function for threedimensional self-organizing feature maps (3D SOM) algorithm and applied them to three-dimensional image compression coding. Experimental results show that the 3D orthogonal cross neighborhood shape and exponential function algorithm have better peak signal to noise ratio (PSNR) and subject quality than others.
\end{abstract}

Keywords—self-organizing maps; three-dimensional image coding; pattern recognition; neighborhood algorithm

\section{INTRODUCTION}

The self-organizing map (SOM) proposed by Kohonen is a highly intelligent information processing method that forms a topologically ordered mapping from the high-dimensional data space to a low-dimensional representation space [1]. It has already found wide applications in such areas as pattern recognition [2]-[4], data mining [5]-[8], automatic control [9][10] and failure detection [11]-[12] and so on.

Traditional SOM network can effectively deal with onedimensional and two-dimensional signal, but can not deal with three-dimensional signal directly. To solve this problem, 3D SOM algorithm has been proposed in recent years. In 3D SOM theory, the neighborhood is one of the core concepts of self-organization mechanism, which reflects the interaction and mutual influence between the winning neuron and the adjacent neurons, and has important affection on the quality of learning and convergence performance of SOM algorithm. For this reason, in order to improve the performance of 3D SOM algorithm, we need to find new three-dimensional neighborhood shape and neighborhood decay function. In this paper, we propose three kinds of neighborhood shapes and two kinds of neighborhood decay functions, and compare the performance difference.

\section{3D SOM ALGORITHM}

The network structure of 3D SOM algorithm is shown in Fig.1. The mapping layer neurons are arranged in a threedimensional structure, the number of rows, columns and layers can take different values, and different three-dimensional network structure usually result in different performance. The shape of three-dimensional neighborhood can have different choice, we usually choose spherical neighborhood, square neighborhood or three-dimensional orthogonal cross

This paper was supported by The Natural Science Foundation of China (No.61261035) and Innovation Project of GUET Graduate Education (No.GDYCSZ201451). neighborhood, algorithm performance varies when selecting different three-dimensional shape of neighborhood. 3D SOM network is the same as ordinary competition network, for each input pattern, there is corresponding winning node in mapping layer, winning nodes represent the most similar pattern, the winning nodes and all nodes in its three-dimensional neighborhood adjust their own weight according to certain rules. When the input pattern changes, there will be a different pattern win through competition. In this way, the network adjusts the network weights through a large number of training samples by means of the self-organizing way. Finally the weight vector space is in accordance with the probability distribution of input patterns, namely the weight vector space can reflect the statistical characteristics of input pattern.

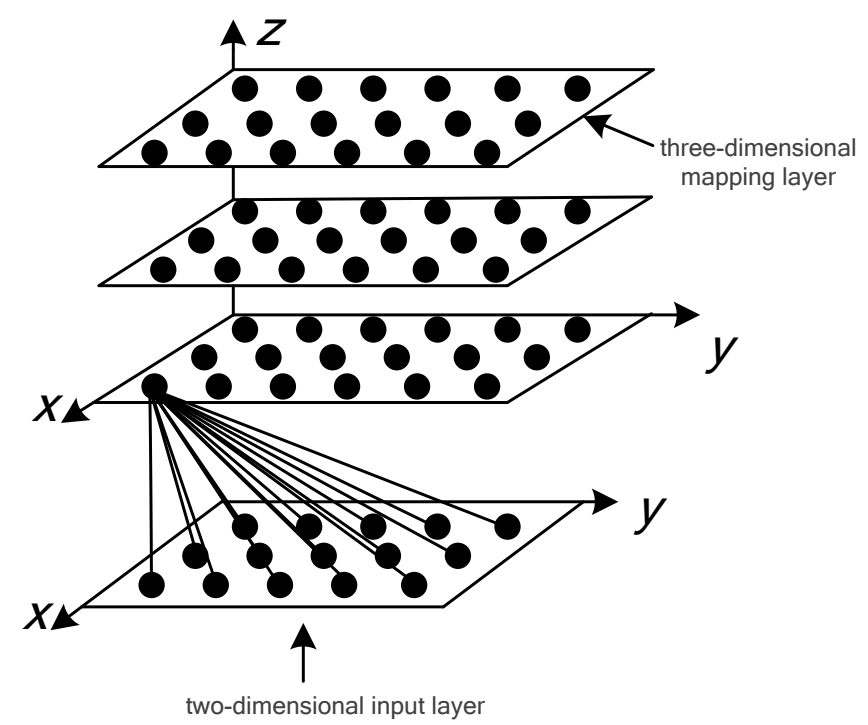

Fig.1 3D SOM network structure

\section{3D SOM NEIGHBORHOOD ALGORITHM}

\section{A. 3D Neighborhood Shape}

The neighborhood shape of 3D SOM is a very important factor for the performance of the pattern library. We propose three kinds of neighborhood shapes, cube neighborhood, sphere neighborhood, 3D orthogonal cross neighborhood. Fig.2 (a) show the cube neighborhood, the length of cube side is twice the radius of neighborhood. Fig.2 (b) show the sphere neighborhood, the radius of sphere is the radius of neighborhood. Fig.2 (c) show the 3D orthogonal cross 
neighborhood, the length in each direction is the radius of neighborhood. The performance of 3D SOM algorithm is different when choosing different neighborhood shape.

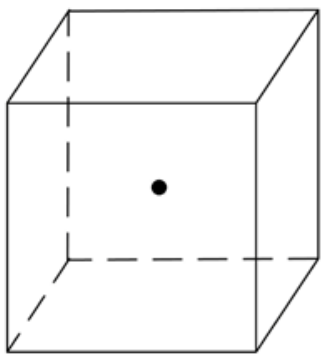

(a) cube neighborhood

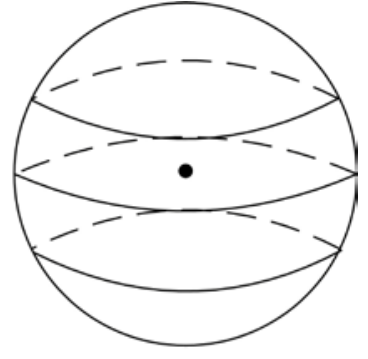

(b) sphere neighborhood

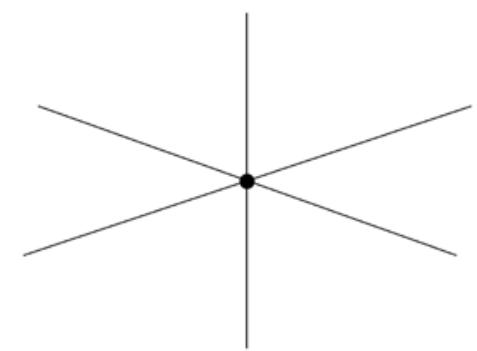

(c) 3D orthogonal cross neighborhood

Fig.2 Three kinds of 3D neighborhood shape

\section{B. 3D Neighborhood Decay Function}

Neighborhood function $N E(t)$ decreases with time $t$. When $t$ is sufficiently large, $N E(t)$ tends to a constant, the SOM network tends to stability. We proposed the linear and exponential functions as neighborhood decay function for 3D SOM algorithm.

(1) Linear function

$$
N E(\mathrm{t})=N E_{\min }+\left(N E_{\max }-N E_{\min }\right) *(T-t) / T
$$

(2) Exponential function

$$
N E(t)=N E_{\min }+N E_{\max } \mathrm{e}^{-\mathrm{t} / T}
$$

$N E_{\max }$ and $N E_{\min }$ are constants determining the range of the neighborhood, and $T$ is a constant determining the decreasing rate. $t$ is the number of iterations. When training begins, $t=0$, $N E=N E_{\max }$, as the training progresses, $t$ increases constantly, $N E$ tend to $N E_{\min }$.

\section{EXPERIMENTAL RESULTS}

We use two testing grayscale stereo images to test our 3D SOM neighborhood algorithm, Tree and Plant. The reconstructed image quality is measured by PSNR, where $R_{\mathrm{PSNR}}=10 \lg \frac{255^{2}}{E_{\mathrm{MSE}}} \mathrm{dB}$, and MSE is the mean square error between the original image and the reconstructed image. Ratio of image compression is calculated by $C_{\mathrm{R}}=\frac{M \times B_{\mathrm{O}}}{B_{\mathrm{C}}}$, where
$M$ is the dimensions of the pattern vector, $\mathrm{B}_{0}$ is bits of the original image pixels, $B_{c}$ is bits of pattern vector class indexes.

In the experiment, the pattern library size is 2048 (compression ratio $C_{\mathrm{R}}=\frac{64 \times 8}{11}=46.5$ ). We use 3D SOM algorithm with different neighborhood shape or neighborhood decay function to compress the disparity of the stereo image, and compare the prediction performance using PSNR between the original image and predicted image. Tab.1 shows PSNR comparison of three neighborhood shapes. It is clear that 3D orthogonal cross neighborhood achieve the best performance. Tab.2 shows PSNR comparison of two neighborhood decay functions. It is clear that exponential function has better than linear function.

TABLE I. PSNR COMPARISON OF THREE NEIGHBORHOOD SHAPES (dB)

\begin{tabular}{|c|c|c|c|}
\hline Neighborhood shape & Cube & Spherical & 3D orthogonal cross \\
\hline Tree & 31.61 & 30.43 & 33.39 \\
\hline Plant & 30.43 & 28.93 & 32.69 \\
\hline
\end{tabular}

TABLE II. PSNR COMPARISON OF TWO NEIGHBORHOOD DECAY FUNCTIONS (dB)

\begin{tabular}{|c|c|c|}
\hline Neighborhood decay function & Linear function & Exponential function \\
\hline Tree & 29.55 & 33.39 \\
\hline Plant & 27.63 & 32.69 \\
\hline
\end{tabular}

\section{References}

[1] Kohonen T.Essentials of the self-organizing map[J].Neural Networks,2013,37:52-65.

[2] Hikawa H, Kaida K. Novel FPGA Implementation of Hand Sign Recognition System With SOM-Hebb Classifier[J]. Circuits and Systems for Video Technology, IEEE Transactions on, 2015, 25(1): 153-166.

[3] Ben Haj Ayech M, Amiri H. Content-based image retrieval in the topic space using SOM and LDA[C]//Control, Engineering \& Information Technology (CEIT), 2015 3rd International Conference on. IEEE, 2015: 1-6.

[4] Kuremoto T, Kobayashi K, Otani T, et al. One dimensional ring type growing SOM with asymmetric neighborhood function and its application to a hand shape instruction learning system[C]//Software Engineering, Artificial Intelligence, Networking and Parallel/Distributed Computing (SNPD), 2014 15th IEEE/ACIS International Conference on. IEEE, 2014: 1-6.

[5] Ma Z, Yang Y, Wang F, et al. The SOM Based Improved K-Means Clustering Collaborative Filtering Algorithm in TV Recommendation System[C]//Advanced Cloud and Big Data (CBD), 2014 Second International Conference on. IEEE, 2014: 288-295.

[6] Sanghvi Y, Gupta H, Doshi H, et al. Comparison of Self organizing maps and Sammon's mapping on agricultural datasets for precision agriculture[C]//Innovations in Information, Embedded and Communication Systems (ICIIECS), 2015 International Conference on. IEEE, 2015: 1-5.

[7] Sapkota N, Karwowski W, Ahram T. Application of Evolving SelfOrganizing Maps for Analysis of Human Adverse Events in the Context of Complex Socioeconomic Infrastructure Interactions[J]. HumanMachine Systems, IEEE Transactions on, 2015, 45:500-509.

[8] George A J, Gopakumar G, Pradhan M, et al. A self-organizing mapharmony search hybrid algorithm for clustering biological data[C]// Signal Processing, Informatics, Communication and Energy Systems (SPICES), 2015 IEEE International Conference onIEEE, 2015. 
[9] Wang C H. Toward a New Task Assignment and Path Evolution (TAPE) for Missile Defense System (MDS) Using Intelligent Adaptive SOM with Recurrent Neural Networks (RNNs)[J]. IEEE Transactions on Cybernetics, 2015, 45:1134-1145.

[10] Honda S, Narita Y, Kida N. Adaptive control for vibration suppression by using self-organization map[C]//Control Conference (ASCC), 2015 10th Asian. IEEE, 2015: 1-5.

[11] Zhai X, Appiah K, Ehsan S, et al. A Method for Detecting Abnormal Program Behaviour on Embedded Devices[J]. IEEE Transactions on Information Forensics \& Security, 2015:1-1.

[12] Minhoe Kim, Souhwan Jung, Minho Park. A Distributed SelfOrganizing Map for DoS attack detection[C]// Ubiquitous and Future Networks (ICUFN), 2015 Seventh International Conference onIEEE, 2015. 